\title{
DYNAMIC FINITE ELEMENT MODELING OF DISLOCATIONS IN A LATERALLY HETEROGENEOUS CRUST
}

\author{
Robert J. Geller, ${ }^{*}$ Gerald A. Frazier, ${ }^{* *}$ \\ and Martin W. MCCANN, JR. ${ }^{* * *}$ \\ *Department of Geophysics, Stanford Uuiversity, Stanford, CA 94305, U.S.A. \\ **Del Mar Technical Associates, P.O. Box 1083, \\ Del Mar, California, U.S.A. \\ ***John A. Blume Earthquake Engineering Center, Stanford University, \\ Stanford, California, U.S.A. \\ (Received May 14, 1979)
}

\begin{abstract}
Elastic wave propagation in a laterally and vertically heterogeneous crust is modeled using the dynamic finite element method (DFEM). By using the elastic representation theorem we replace dislocation sources with arbitrary orientation by their equivalent body forces. It is then possible to model a dipping fault numerically using a regular rectangular grid with either finite element or finite difference methods. Our DFEM fault model is verified by comparison with analytic dislocation solutions. We then present a simple example of the effects of crustal lateral heterogeneity and rupture complexity on the resulting displacements.
\end{abstract}

\section{Introduction}

Many investigators have used whole space or half space models in studies of near field seismograms from earthquakes and explosions. It is desirable to determine if the effect of a heterogeneous medium on near field wave forms can be neglected. In particular, whole space models have been used in many studies to infer the spatial and temporal distribution of faulting. In this paper we examine the effect of a laterally varying crustal model in the near field.

Numerical methods must be used for near field wave propagation problems in laterally and vertically heterogeneous media, because general analytic solutions do not exist. Dynamic finite element and finite difference methods are the two obvious choices, since both can treat arbitrarily varying media. For this study, we use the dynamic finite element method (DFEM) described by Frazier et al. (1973) and Frazler and Petersen (1974). Previous DFEM faulting studies (e.g. MCCowan et al., 1977; ARCHUleta and Frazier, 1978) have modeled faults with prescribed stress 
drop or prestress. In this paper we study faults on which the slip is specified as a function of space and time (a shear dislocation). Such models are useful for determining the ground motion which a particular faulting history would cause in a heterogeneous medium. In the following sections we develop the methods of implementing DFEM dislocation sources, verify the numerical results by comparison with analytic solutions and finally demonstrate the effect of crustal structure on seismic waveforms. We present only plane strain results due to computational bounds.

\section{DFEM Modeling of Dislocations}

The theoretical basis of the finite element method is summarized by STrANG and FIX (1973) and Zienkiewicz (1971). Essentially one discretizes a continuous system to obtain a matrix equation in terms of displacements at nodal points. For linear elasticity, the equation is

$$
[K]\{U\}+[C] \frac{\partial}{\partial t}\{U\}+[M] \frac{\partial^{2}}{\partial t^{2}}\{U\}=\{F\}
$$

where $[K]$ is the stiffness matrix, $[M]$ the mass matrix, $\{U\}$ the vector of nodal displacements and $\{F\}$ is the vector of applied forces. [C] is a matrix which is added to introduce artificial numerical damping. Frazler et al. (1973) show that [C] can be used for damping which is proportional to frequency squared; thus high frequencies (relative to grid size and time step), which cannot be calculated accurately in any case, are damped.

The representation theorem of DE HOOP (1958) and BURRIDGE and KNopoff (1964) allows one to replace a dislocation by an equivalent distribution of force couples and dipoles. For purposes of the DFEM dislocation calculation, the force systems can then be discretized to give forces acting on the nodal points, and having the same seismic moment. (This amounts to replacing a derivative by spatial differencing.) The effective location of the discretized sources will be at the center of each element, regardless of where the fault actually passes through the element. For a plane strain problem in the $x_{1}-x_{2}$ plane, we let $\Gamma_{i j}(i, j=1,2)$ denote the two dimensional Green's tensor. If $\theta$ is fault orientation (counterclockwise from the $x_{1}$ axis), $\mu$ is rigidity and $D(\xi, t)$ is dislocation history along the fault (right lateral is positive), then the displacement anywhere in the medium, from the representation theorem (see Geller, 1974) is

$$
u_{i}(x, t)=-\mu \int_{W}\left\{\cos 2 \theta\left(\Gamma_{i 1,2}+\Gamma_{i 2,1}\right)+\sin 2 \theta\left(\Gamma_{i 2,2}-\Gamma_{i 1,1}\right)\right\} D(\xi, t) d \xi .
$$

Where $\boldsymbol{x}$ is receiver position and $\boldsymbol{\xi}$ is position along the fault. (There is 


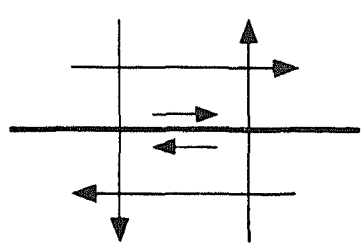

(a)

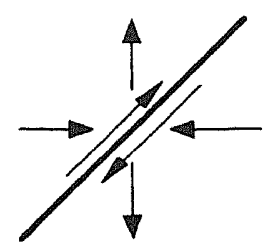

(b)

Fig. 1. (a) Force system corresponding to $-\left(\Gamma_{i 1,2}+\Gamma_{i 2,1}\right)$, right lateral faulting on the plane with $\theta=0^{\circ}$. (b) Force system corresponding to $-\left(\Gamma_{i 2,2}-\Gamma_{i 1,1}\right)$, right lateral faulting on the plane with $\theta=45^{\circ}$.

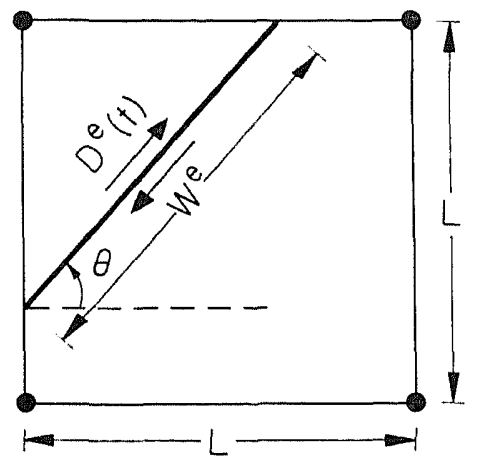

Fig. 2. A segment of a continuous fault with length $W^{e}$ in the square element. We replace the continuous fault by discrete body forces on the nodes representing a source with the same moment and orientation acting at the center of the element.

a negative sign in front of the integral because the partial derivatives are with respect to $\boldsymbol{x}$, not $\boldsymbol{\xi}$.) The systems of force couples and dipoles referred to by (2) are shown in Fig. 1.

The moment of a fault segment which $W^{e}$ long and have average dislocation $D^{e}(t)$ is

$$
M_{0}{ }^{e}(t)=\mu^{e} W^{e} D^{c}(t) .
$$

The superscript refers to the element through which the fault segment extends, shown in Fig. 2.

We introduce two systems of vectors acting on the nodes of an element which contain a fault segment, $\left\{\right.$ TYPE $\left.1_{i}\right\}$ and $\left\{\right.$ TYPE $2_{i}$, shown in Fig. 3. (These force systems are much easier to show graphically than to define symbolically). \{TYPE $\left.1_{i}\right\}$ is the discretized equivalent body force system for either a horizontal right lateral fault or its conjugate system. $\left\{\right.$ TYPE $\left.2_{i}\right\}$ is the discretized equivalent body force system for a right lateral fault striking $45^{\circ}$ counterclockwise from the horizontal. 


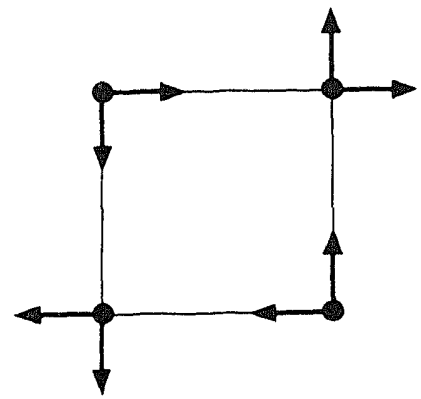

(a)

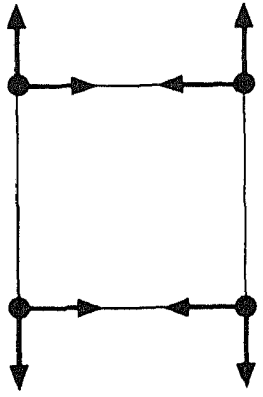

(b)

Fig. 3. (a) \{TYPE $1_{i}$ \}. A system of discrete vectors with each component having magnitude one and the indicated direction. \{TYPE $1_{i}$ \} is used for the $\theta=0^{\circ}$ fault. (b) $\{$ TYPE $2 i\}$. Discrete vector for the $\theta=45^{\circ}$ fault.

Thus $\left\{\right.$ TYPE $\left.1_{i}\right\}$ and $\left\{\right.$ TYPE $\left.2_{i}\right\}$ are the DFEM analog of equivalent body forces for dislocations, and replace, respectively, the first and second Green's function terms in (2). Therefore, just as in the continuous case, we can replace a dislocation at an arbitrary angle by a linear combination of $\left\{\right.$ TYPE $\left.1_{i}\right\}$ and $\left\{\right.$ TYPE $\left.2_{i}\right\}$ given by

$$
\left\{F_{i}\right\}=\left\{\left(\text { TYPE } 1_{i}\right) \cos 2 \theta+\left(\text { TYPE } 2_{i}\right) \sin 2 \theta\right\}\left(\iota^{e} D^{e}(t) W^{e}\right) /(2 L) .
$$

$L$ is the length of the side of a square element. The force couples and dipoles in (4) give the correct moment (3). This method for discretizing sources is somewhat similar to one given by ABoudr (1971) for a finite difference method.

In the calculations for this study we use a completely equivalent procedure which is easier to implement. Instead of imposing the forces (4), we temporarily modify the nodal displacements when we calculate strains within an element.

$$
\left\{U_{i}^{e}\right\} \leftarrow\left\{U_{i}^{e}\right\}-\left\{\left(\text { TYPE } 1_{i}\right) \cos 2 \theta+\left(\text { TYPE } 2_{i}\right) \sin 2 \theta\right\}\left(D^{e}(t) W^{e}\right) /(2 L) .
$$

The DFEM will then calculate the stresses caused by the imposed strains, the restoring forces and finally, the accelerations.

\section{Comparison to Analytic Results}

We verify the DFEM dislocation source by comparison with analytic solutions for a plane strain dislocation in a homogeneous whole space, given by GeLler (1974). Both analytic and DFEM results were obtained for the geometry shown in Fig. 4. The dislocation propagated upward with a rupture velocity of $2.85 \mathrm{~km} / \mathrm{sec}$. The ramp time function had a rise time of $0.6 \mathrm{sec}$ and final offset of $100 \mathrm{~cm}$. Material constants were 


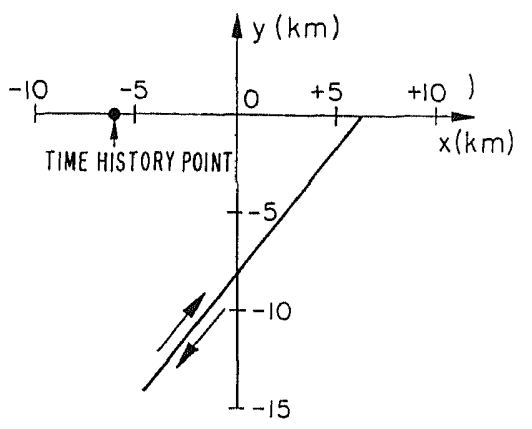

Fig. 4. Fault and receiver geometry for the comparison of the analytic dislocation solution to the DFEM whole space and half space calculations.

$\alpha=5.7 \mathrm{~km} / \mathrm{sec}$ and $\beta=3.1 \mathrm{~km} / \mathrm{sec}$. A (dimensionless) frequency dependent damping coefficient of 0.15 , and a time step of $0.2 \mathrm{sec}$ are used for this example.

We make a whole space DFEM calculation for direct comparison with the analytic solutions. We also make a half space DFEM run to estimate the accuracy of the usual procedure of doubling the whole space displacements to approximate the effects of the free surface. For both runs the DFEM elements are squares with $2 \mathrm{~km}$ sides. The DFEM whole space grid has 30 elements horizontally (from $x=-30$ to $x=30$ ) and 30 vertically (from $y=-40$ to $y=20$ ). The upper 10 elements are omitted for the DFEM halfspace run. For all DFEM calculations in this paper we use results only at times before reflections from the boundary of the numerical grid arrive at observation points.

Figures 5 and 6 show comparisons of analytic, DFEM whole space and DFEM half space results. We divide the half space results by two (rather than double the whole space results), permitting the accuracy of the free surface approximation to be determined by inspection. Displacements at the time history point (shown in Fig. 4) are plotted in Fig. 5. The analytic and DFEM whole space solutions agree very well. The DFEM halfspace solution, although initially in agreement with the whole space, differs markedly after 10 seconds. Figure 6 is a "snapshot" of displacements along the free surface five seconds after the initial faulting. All three solutions are basically in agreement, although the analytic solution, which contains the high frequencies, has greater amplitudes. The discontinuous slope of the analytic curves in Figs. 5 and 6 results from plotting the analytic solutions at the same spatial and temporal intervals as the DFEM results.

To investigate the frequency response of the DFEM results, we conducted a second test in which we compared the DFEM whole space results to the analytic solutions. We used a fault running from $(+5,-0.5)$ to 

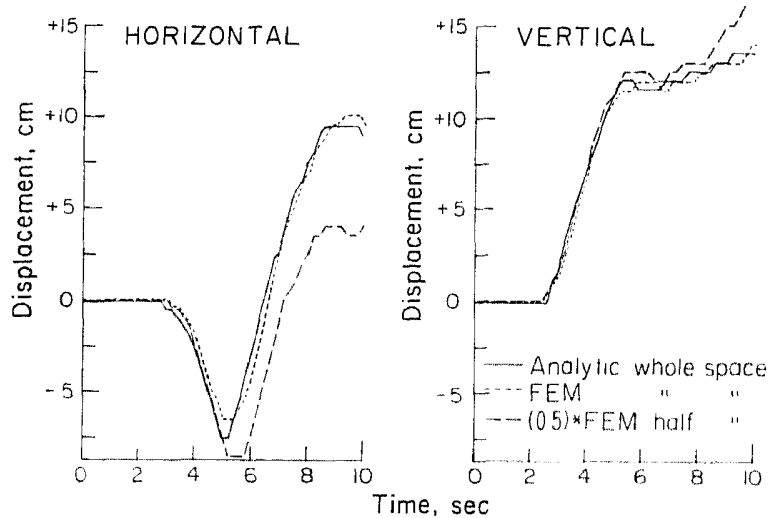

Fig. 5. Displacements as a function of time at the time history point shown in Fig. 4.

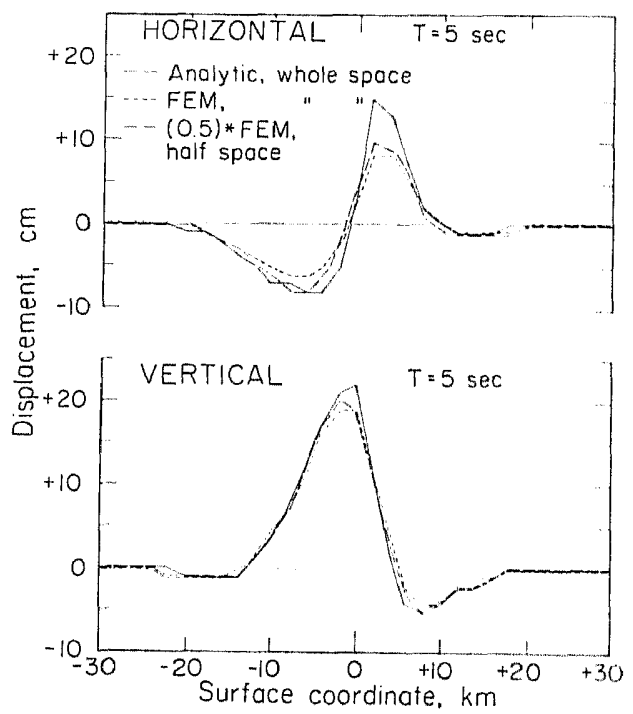

Fig. 6. A "snapshot" of displacements along the "free surface" $(y=0)$ shown in Fig. 4.

$(-5,-0.5)$, with right lateral slip. The dislocation propagated to the right with a rupture velocity of $2.3 \mathrm{~km} / \mathrm{sec}$. The dislocation rise time had a value of $3.0 \mathrm{sec}$. and final offset of $100 \mathrm{~cm}$. Material properties were $\alpha=6.0 \mathrm{~km} / \mathrm{sec}$ and $\beta=3.5 \mathrm{~km} / \mathrm{sec}$. A (dimensionless) frequency dependent dumping coefficient of 0.30 is used in this example.

We used two different grid sizes, $1 \mathrm{~km}$ and $2 \mathrm{~km}$, to study the increase in accuracy from the finer mesh. In order to present the results more clearly, both the displacements and velocities are shown. The velocities 

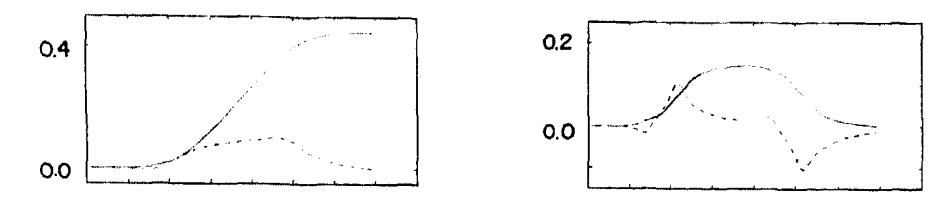

ANALYTIC
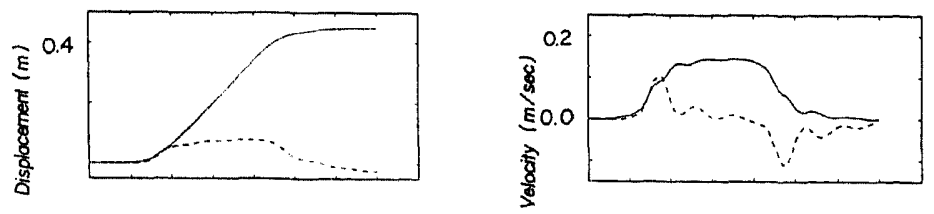

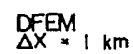
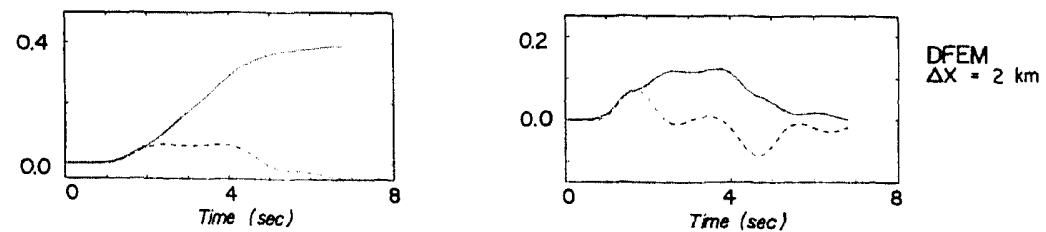

Fig. 7. Displacements and velocities for the second test case, at $(0,0)$, which is $1 / 2 \mathrm{~km}$ above the fault. (Solid line is horizontal component; dashed is vertical.)

have a higher predominant frequency than the displacements, and thus are more sensitive to the limitations imposed by the coarseness of the numerical grid. The DFEM grid has dimensions in the $x$-direction from $x=-30$ to $x=30$ and in the $y$-direction from $y=-40$ to $y=20$. The time steps used are $0.2 \mathrm{sec}$ for the $2 \mathrm{~km}$ grid and $0.1 \mathrm{sec}$ for the $1 \mathrm{~km}$ grid.

Figure 7 shows time histories at $x=0, y=0$, located $1 / 2 \mathrm{~km}$ above the center of the fault. The displacements compare quite well, because the low frequencies predominate. The velocities are more revealing of the limitations in frequency resolution. The DFEM calculations successfully replicate the overall features of the analytic solution, but are lacking in resolution at higher frequencies. The $1 \mathrm{~km}$ DFEM calculations are less sharp than the analytic results and the $2 \mathrm{~km}$ results are still more smeared out. This is basically what is expected since, as a general rule of thumb, finite element or finite difference calculations are accurate only at frequencies for which there are more than 6 to 10 elements per wavelength. Figure 8 , the time histories at $x=-10, y=0$, shows basically similar results-the agreement between the analytic and numerical results is excellent at low frequencies, but the DFEM results include accurate information only at frequencies with a sufficient number of elements per wavelength.

In Fig. 9 snapshots of displacements and velocity along the free surface three seconds after the initiation of faulting are given. All three DFEM results again show good agreement with the analytic results for velocities as well as displacements. The velocity trances do however show the major differences more clearly. As expected, the smaller element size is 

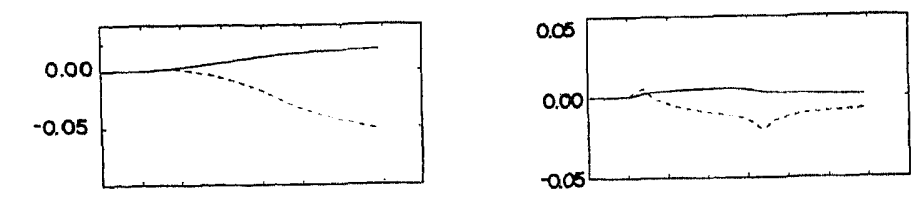

ANALYTIC
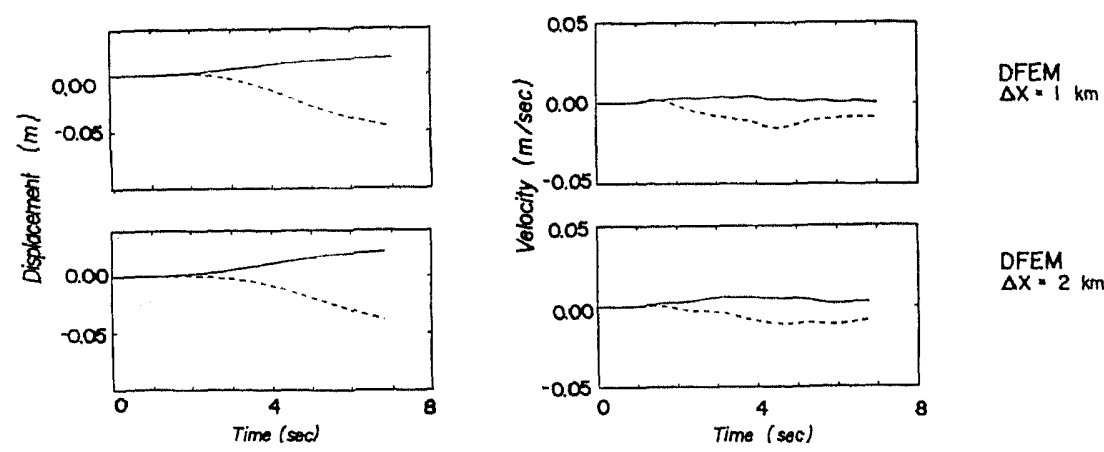

DFEM

$\Delta x=2 \mathrm{~km}$

Fig. 8. Displacements and velocities for the second test case, at $(-10,0)$. (Solid line is horizontal component; dashed is vertical.)
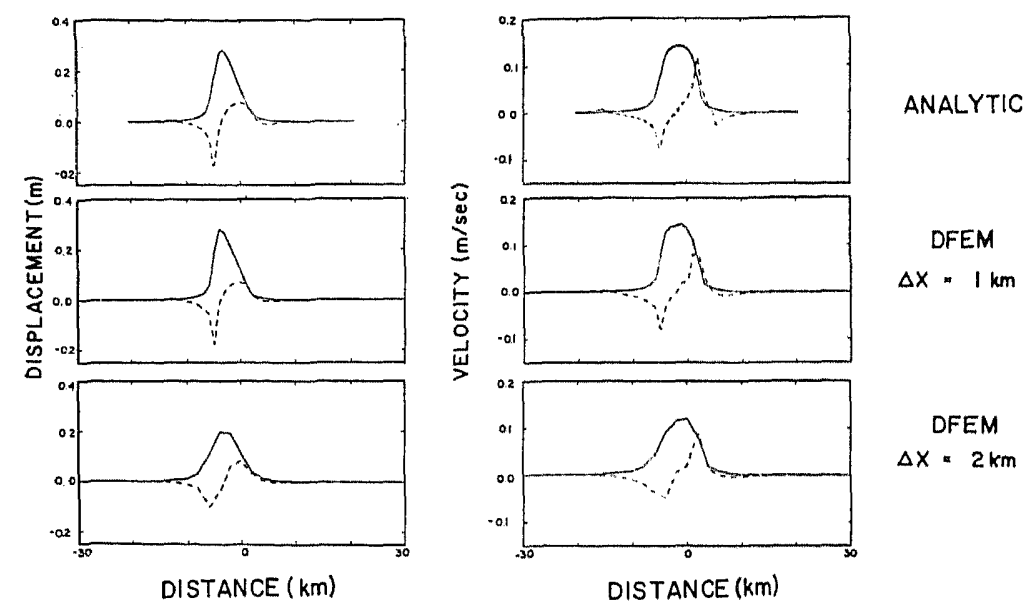

Fig. 9. "Snapshot" of displacements along the line $y=0$ (for the second test) case, at $t=3 \mathrm{sec}$. (Solid line is horizontal component; dashed is vertical.)

able to more accurately model the higher frequencies. In Fig. 10 Fourier amplitude spectra are shown for the displacement snapshots in Fig. 9. They show, more quantitatively, the resolution limits of the DFEM results.

\section{DFEM Nearfield Calculations}

We construct a simple model chosen for its schematic resemblance to the Feb. 9, 1971 San Fernando earthquake. Using this model, we 


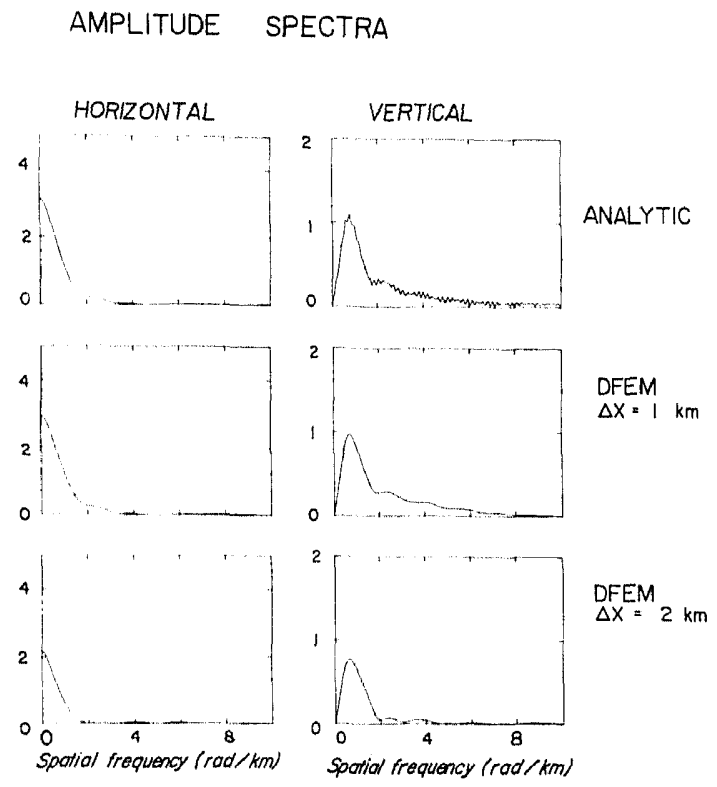

Fig. 10. Wavenumber spectra of the displacements in Fig. 9.

(The horizontal scale is labeled incorrectly. The numbers should be divided by ten and the units are cycles $/ \mathrm{km}$.)

examine the relative effects of source complexity and crustal structure in affecting the character of long period waveforms.

We use two models of crustal structure. One, a complex crustal structure adapted from refraction data of KANAMORI and HADLEY (1975), is shown in Fig. 11. The layer boundaries are moved to coincide with element boundaries, and a layer of low velocity material is used for the top of the "southern" (left in Fig. 11) half, to simulate the sedimentary San Fernando Valley. Shear wave velocities in all but the sedimentary layer are obtained by assuming $\alpha=\sqrt{ } 3 \beta$. The other crustal model was a uniform half space with $\alpha=5.7 \mathrm{~km} / \mathrm{sec}$ and $\beta=3.1 \mathrm{~km} / \mathrm{sec}$. Because of the relatively long periods we examine, the effect of surface topography (BOore, 1973) is negligible.

We use the fault geometry shown in Fig. 11: a thrust fault with $50^{\circ} \mathrm{dip}$, extending to a depth of $11 \mathrm{~km}$. For all of our DFEM runs, we use a propagating ramp rupture with velocity of $2.85 \mathrm{~km} / \mathrm{sec}$ and rise time of $0.6 \mathrm{sec}$. The DFEM elements are squares with $2 \mathrm{~km}$ sides in a 50 (horizontal) $\times 30$ (vertical) grid. We use two dislocation models, shown in Fig. 12. The "complex fault" is a simplified version of ALEWINE's (1974) static dislocation profile and is used for its suggestive resemblance, not for exact comparison to the details of San Fernando. For all our 


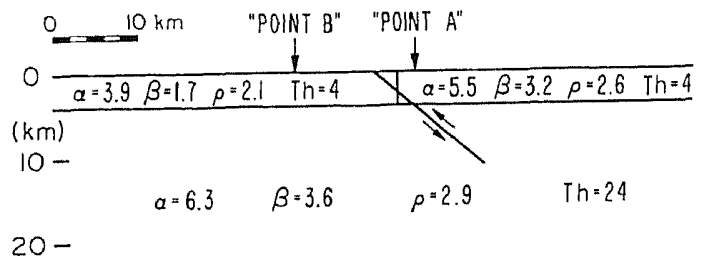

$\begin{array}{ccccc}30= & \alpha=6.8 & \beta=3.9 & \rho=3.0 & T h=4 \\ 40- & \alpha=7.8 & \beta=4.5 & \rho=3.3 & T h=\infty \\ & & & & \downarrow\end{array}$

Fig. 11. Crustal structure used for the DFEM earthquake simulation. $\alpha$ is compressional velocity, $\beta$ is shear velocity, $\rho$ is density and $T h$ is layer thickness. Fault and receiver geometry is shown.

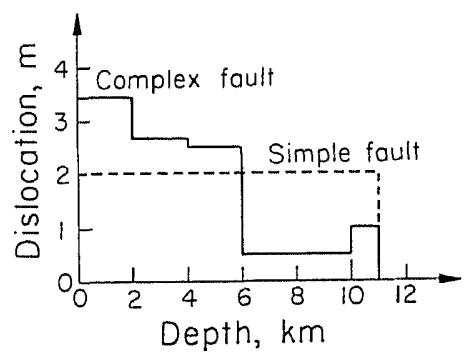

Fig. 12. Complex and simple dislocation profiles.

calculations we display the displacements at the two points shown in Fig. 11. Points $A$ and $B$ are the same distance from the fault in Fig. 11 as some instruments (e.g. Pacoima Dam and Holiday Inn for the San Fernando earthquake) whose records have been modeled with a whole space by some investigators.

The purpose of our DFEM runs is to determine the relative importance of details of the source dislocation history and crustal structure in affecting the character of waveforms. We find that, for the cases we considered, crustal structure has far more effect on the waveforms than the source. We make four runs, for all four combinations of simple vs. complex fault and complex crustal structure vs. uniform half space. The displacements calculated for each of the four models are plotted in Figs. 13 and 14.

Displacements at Point A, given in Fig. 13 show the effect of crustal structure is more important than the source effects. Traces I and III in Fig. 13 are half space calculations for the simple and complex fault respectively, while Traces II and IV are for the complex crustal structure. 


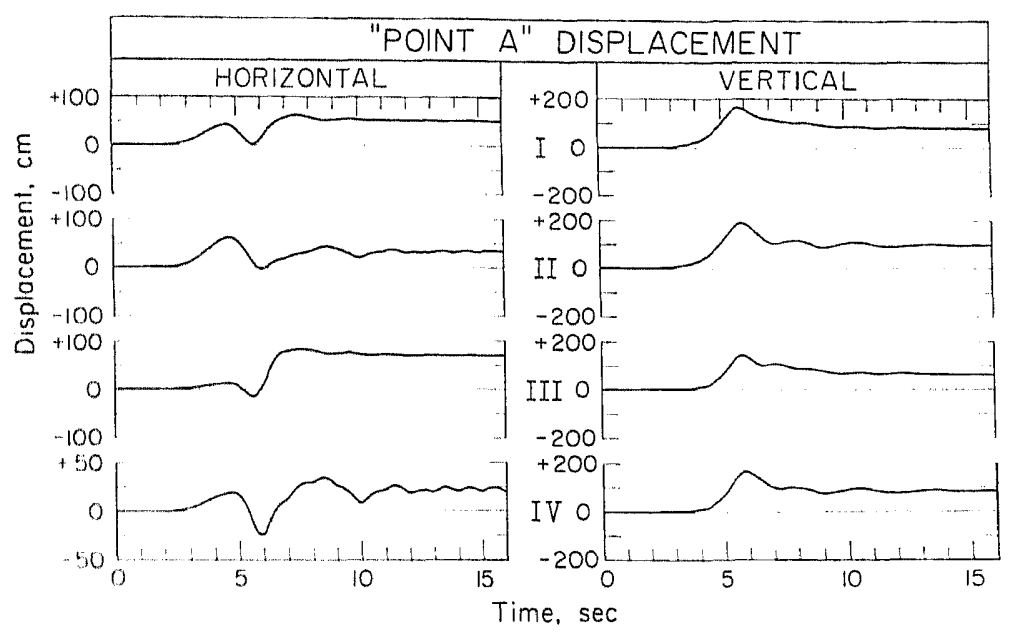

I Half space, simple fault.

III Half space, complex fault.

II Structure, " "

IV Structure,

Fig. 13. Displacements at Point $\mathrm{A}$ for the four model calculations. Positive displacements are to the "south" (Left) and up. Roman numerals I to IV correspond to the four cases explained in the legend. (The one second oscillations in the later part of some traces is due to the coarse, $2 \mathrm{~km}$, grid size.)

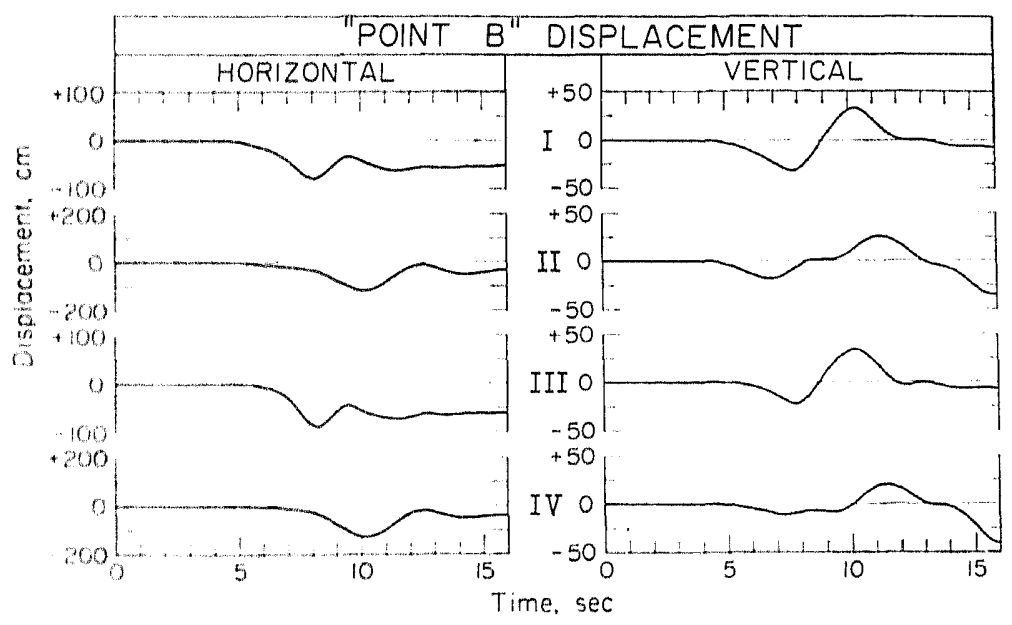

I Holf spoce, simple fault.

III Holf space, complex fault.

II Structure,

IV Structure.

Fig. 14. Displacements at Point B. Sign and labeling conventions are identical to Fig. 9. 
The horizontal components for models with the same structure have much more in common than those with the same source. Varying the source and structure has relatively little effect on the vertical components, suggesting that the horizontal components are altered more by the impedance mismatch at the vertical boundary.

The Point B displacements, given in Fig. 14 show a great similarity between models with the same crustal structure. The shapes of both the horizontal and vertical waveforms for the same structure are nearly identical, irrespective of which source was used. The only significant exception to this is the vertical component of Trace IV, which has a smaller first arrival than Trace II, caused by the smaller initial dislocation at depth. The horizontal amplitudes above the sedimentary layer are nearly twice as large as the half space amplitudes. Also, note the "stretched out" nature of the numerical seismograms for the sedimentary layer, caused by dispersive propagation in the layered medium.

\section{Discussion}

Different models of crustal structure can affect the character of long period waveforms. For either finite element or finite difference calculations, our method makes it possible to model a dipping dislocation source in a laterally and vertically heterogeneous medium, using a rectangular grid.

We thank Ralph Archuleta, David Boore, David Harkrider, Don Helmberger, Hiroo Kanamori, Dan Kosloff and Seth Stein for helpful discussions and for critically reading the manuscript. This research was supported by the National Science Foundation under grant EAR 78-03653. Early portions of the work were completed while the authors were at the Seismological Laboratory, California Institute of Technology (R.J.G.) and the Institute of Geophysics and Planetary Physics, University of California, San Diego (G.A.F).

\section{REFERENCES}

ABoudi, J., Numerical simulation of seismic sources, Geophysics, 36, 810-821, 1971.

Alewine, R.W. III, Application of linear inversion theory toward the estimation of seismic source parameters, Thesis, California Inst. of Technology, 1974.

Archuleta, R. J. and G. A. FrazXer, Three dimensional numerical simulations of dynamic faulting in a halfspace, Bull. Seismol. Soc. Am., 68, 541-572, 1978.

Boore, D.M., The effect of simple topography on seismic waves: implications for the accelerations recorded at Pacoima Dam, San Fernando Valley, Calif., Bull. Seismol. Soc. Am., 63, 1603-1609, 1973.

BURRIDGE, R. and L. KNOPOFF, Body force equivalents for seismic dislocations, Bull. Seismol. Soc. Am., 54, 1875-1888, 1964. 
DE Hoop, A. T., Representation theorems for the displacement in an elastic solid and their application to elastodynamic diffraction theory, Thesis, Technische Hogeschool, Delft, 1958.

Frazier, G. A. and C. M. Petersen, 3-D stress wave code for the Illiac IV, Systemes, Science and Software Report SSS-R-74-2103, La Jolla, California, 1974.

Frazire, G. A., J. H. Alexander, and C. M. Peterson, 3-D seismic code for the Illiac IV, Systems, Science and Software Report SSS-R-73-1506, La Jolla, California, 1973.

GiLl.:R, R. J., Representation theorems for an infinite shear fault, Geophys. J.R. Astron. Soc., 39, 123-131, 1974.

KANAMORI, H. and D. HADLi:y, Crustal structure and temporal velocity change in Southern California, Pure Appl. Geophys., 113, 257-280, 1975.

Mc.Cowan, D. W., P. Glover, and S.S. Alexander, A static and dynamic finite element analysis of the 1971 San Fernando, California, Earthquake, Geophys. J.R. Astron. Soc., 48, 163-185, 1977.

Strang, G. and G. J. Fix, An Analysis of the Finite Element Method, Prentice-Hall, Englewood Clifts, N.J., 1973.

ZniNkrewicz, O.C., The Finite Element Method in Engineering Science, 2nd edition, McGraw-Hill, London, 1971. 